\title{
A Herpesvirus saimiri-based vector expressing TRAIL induces cell death in human carcinoma cell lines and multicellular spheroid cultures
}

\author{
SUSAN J. TURRELL ${ }^{1,2}$, STUART A. MACNAB ${ }^{1,2}$, AILSA ROSE $^{3}$, \\ ALAN A. MELCHER ${ }^{3}$ and ADRIAN WHITEHOUSE ${ }^{1,2}$ \\ ${ }^{1}$ Institute of Molecular and Cellular Biology, Faculty of Biological Sciences, ${ }^{2}$ Astbury Centre for \\ Structural Molecular Biology, University of Leeds, Leeds, LS2 9JT; ${ }^{3}$ Cancer Research UK, \\ Leeds Institute of Molecular Medicine, St. James' University Hospital, Leeds, LS9 7TF, UK
}

Received December 1, 2011; Accepted January 5, 2012

DOI: 10.3892/ijo.2012.1394

\begin{abstract}
Herpesvirus saimiri (HVS) is capable of infecting a range of human carcinoma cell types with high efficiency and the viral genome persists as high copy number, circular, non-integrated episomes which segregate to progeny upon cell division. This allows HVS-based vectors to stably transduce a dividing cell population and provide sustained transgene expression for an extended period of time both in vitro and in vivo. Moreover, the insertion of a bacterial artificial chromosome cassette into the HVS genome simplifies the incorporation of large amounts of heterologous DNA for gene delivery. Herein we have produced a recombinant HVS-based vector containing full-length human TRAIL under the control of the $\alpha$-survivin promoter, and subsequently challenged a variety of cancer cell lines with this vector. The TRAIL transgene was expressed in infected colorectal SW480 cells, causing considerable apoptosis induction. Apoptosis was also observed when several other cancer cell lines derived from different tissues were infected. Moreover, co-treatment with Jak inhibitor AG490 led to the disruption of spheroid cultures grown from the melanoma Mel888 line. These data suggest that an HVS gene therapy vector expressing TRAIL could be an effective treatment against cancer.
\end{abstract}

\section{Introduction}

Herpesvirus saimiri (HVS) is a $\gamma-2$ herpesvirus originally isolated from the T-lymphocytes of the squirrel monkey (Saimiri sciureus) where it causes an asymptomatic infection (1). HVS possesses a number of characteristics which make it a promising gene delivery vector $(2,3)$. This potential was first highlighted

Correspondence to: Dr Adrian Whitehouse, Institute of Molecular and Cellular Biology, Faculty of Biological Sciences, University of Leeds, Leeds, LS2 9JT, UK

E-mail: a.whitehouse@leeds.ac.uk

Key words: Herpesvirus saimiri, TRAIL, gene therapy over two decades ago in an early preclinical gene therapy trial. A non-transforming replication competent HVS vector expressing the bovine growth hormone (bGH) was experimentally infected into New World primates. Results demonstrated that the primates produced circulating bGH and developed immunoglobulin $\mathrm{G}$ antibodies directed against bGH (4). Subsequent studies have also demonstrated properties of HVS that make it wellequipped for gene therapy applications. Its large genome can transport multiple genes to a target cell (5). Furthermore, HVS can efficiently infect and establish a latent infection in a variety of human cell lines, including carcinoma cells and haematopoietic cells, enabling long-term transgene expression (6-8). The viral genome exists as a stable circular episome separate from host DNA, reducing the risk of insertional mutagenesis and gene silencing by epigenetic mechanisms. HVS can also persist in growing tissues, as the episomal DNA is able to replicate during latency, and is transferred to daughter cells upon cell division via the ORF73 episomal maintenance protein (9-11). Moreover, the virus has also been shown to efficiently infect and persist in three-dimensional multicellular spheroid cultures, a threedimensional cell culture system that closely resembles a tumour, in addition to tumour xenografts in vivo (12-14). Recently, further development in HVS-based vectors include the generation of an HVS amplicon system to increase transgene capacity (15), and the potential of HVS-based vectors for cancer therapy has been highlighted by the use of an HVS-vector expressing shRNAs against endothelin-converting enzyme-1 to prostate cancer cells, which led to the reduction in migration of these cells (16) and reduced proliferation of colorectal cancer cells upon the expression of the adenomatous polyposis coli tumour suppressor gene (17). Moreover, the ability of HVS to transform human T cells has led to the development of HVS as a potential episomal vector for adoptive immunotherapy of infectious and malignant diseases (18-20). These findings make HVS-based vectors a potential and attractive anti-cancer therapeutic.

A desirable transgene for gene therapy directed at cancer is tumour necrosis factor-related apoptosis inducing ligand (TRAIL) or Apo2 ligand (Apo2L) (21). TRAIL is a cancerspecific cytokine which induces apoptosis upon binding death receptors DR4 and DR5 expressed on the surface of cancer 
cells $(22,23)$. Death receptor activation stimulates the extrinsic apoptosis pathway, however this cascade may be abnormally regulated in cancer cells. Several cancers (pancreatic, melanoma, neuroblastoma) are resistant to TRAIL signalling due to mutations in the apoptotic pathways downstream of death receptors (24). Resistant cells can be sensitised to TRAIL by manipulating anti-apoptotic proteins and components of cell signalling pathways $(25,26)$. X-linked inhibitor of apoptosis (XIAP) and FLICE inhibitor protein c-FLIP have been implicated in TRAIL resistance, and blocking their action can enhance TRAIL-induced apoptosis $(27,28)$. These findings and others have given renewed enthusiasm to TRAIL-mediated therapy.

In this study we have constructed a recombinant HVS vector containing full-length human TRAIL under the control of the $\alpha$-survivin promoter, and subsequently challenged a variety of cancer cell lines with this vector. The TRAIL transgene was expressed in infected colorectal SW480 cells, causing considerable apoptosis induction. Apoptosis was also observed when several other cancer cell lines derived from different tissues were infected. Moreover, co-treatment with Jak inhibitor AG490 led to the disruption of spheroid cultures grown from the melanoma Mel888 line. These data suggest that an HVS gene therapy vector expressing TRAIL could be an effective treatment against cancer.

\section{Materials and methods}

Tissue culture. Human cancer cell lines, owl monkey kidney cells and HEK 293T cells were maintained in Dulbecco's modified Eagle's medium (D-MEM) (Invitrogen) supplemented with $5 \mathrm{U} / \mathrm{ml}$ penicillin, $5 \mathrm{U} / \mathrm{ml}$ streptomycin and $10 \%$ heat-inactivated fetal calf serum (FCS). CaSki cells were maintained in RPMI (Invitrogen) with the same antibiotics and FCS. Cells were stored at $37^{\circ} \mathrm{C}$ in a humidified atmosphere with $5 \%$ $\mathrm{CO}_{2}$. The spheroid cultures of the Mel888 cell line were grown in non-coated T75 flasks, where $2 \%$ sterile agar was used to coat the bottom of the flask. Spheroid cultures took $\sim 2$ weeks to form floating masses of $\sim 0.5-1.0 \mathrm{~mm}$.

Construction of HVS $\Delta 71-73$ TRAIL vector. The HVS $\Delta 71-73$ BAC construct is described elsewhere (9). It is based on a bacterial artificial chromosome (BAC) with the BAC elements from the F-plasmid cloned into open reading frame (ORF) 15 of HVS strain A11 S4 (29,30). This insert also contains cassettes for hygromycin phosphotransferase $\left(\mathrm{Hyg}^{\mathrm{R}}\right)$ and dsRed, as well as a unique I-PpoI restriction site to allow conventional cloning into the $160 \mathrm{~kb}$ viral genome. The pShuttle link 2 vector has been described (29). This plasmid contains 2 I-PpoI sites flanking a multiple cloning site. The pTRAIL vector is a custom plasmid from Invivogen, containing the full length human TRAIL gene under the control of the human $\alpha$-survivin promoter. The TRAIL expression cassette from pTRAIL was inserted into pShuttle link 2 via an EcoRI/SalI excision/ligation. The resulting plasmid pSh-TRAIL 2 was digested with I-PpoI to liberate the TRAIL expression cassette, which was then inserted into the I-PpoI site of HVS $\Delta 71-73$. HVS DNA was stored at $4^{\circ} \mathrm{C}$ in TE buffer.

Virus production and infection. Infectious viral particles were produced by transfection of HVS BAC DNA into permissive OMK cells. Transfections were carried out with Lipofectamine 2000 (Invitrogen), in serum-free media. Viral BAC DNA $(2 \mu \mathrm{g})$ was transfected into one well of a confluent 6-well plate of cells with $10 \mu \mathrm{l}$ of the transfection agent according to the manufacturer's protocol. After 6-h incubation, the serum-free media was replaced with $5 \%$ media. Viral growth was monitored by visualising dsRed-positive plaques in the cell monolayer. When the transfected cells were fully lysed, the media was transferred to a confluent T75 of OMK cells (with 5\% media) to scale up virus production. To quantify viral titre, plaque assays were performed $48 \mathrm{~h}$ post-infection, using the permissive OMK cell line. Viral stocks were usually $\sim 1 \times 10^{6}$ infectious particles per $\mathrm{ml}$. Infections were carried out with $1 \times 10^{6}$ cells and viral titres ranging from m.o.i 0.1 to 2. Cells were then cultured in 5\% FCS, and $5 \mathrm{U} / \mathrm{ml}$ penicillin and streptomycin at $37^{\circ} \mathrm{C}$ and $5 \% \mathrm{CO}_{2}$.

Pulse field gel electrophoresis. To analyse restriction digests of viral genomes, $1.2 \%$ agarose gels were made with pulse field electrophoresis grade agarose (Sigma) and 0.5X TBE buffer (0.0225 M Tris-borate, 0.001 M EDTA). A Bio-Rad pulse field gel electrophoresis (PFGE) tank was filled with $0.5 \mathrm{X}$ TBE which was pre-cooled to $15.5^{\circ} \mathrm{C}$ using a Bio-Rad Model 100 mini chiller unit, and circulated with a variable speed pump. The cooling unit and pump were turned off for gel loading. Midrange PFG Marker II (New England BioLabs) was used along with Lambda DNA HindIII Digest (New England BioLabs) to compare sizes of DNA fragments. The CHEF DR II drive module was programmed with the following conditions; $6 \mathrm{~V}$, 12.5 h, Initial SW 2.0, Final SW 16.0. Samples were allowed to run into the gel for $20 \mathrm{~min}$, after which the cooling unit and pump were switched on. Subsequently to electrophoresis, gels were stained using $200 \mathrm{ml}$ of $0.1 \mu \mathrm{g} / \mathrm{ml}$ ethidium bromide (Sigma) in 0.5X TBE buffer.

$R T-q P C R$. Experiments were performed in triplicate. Viruses at m.o.i 1 were used to infect SW480 cells $~ 70 \%$ confluent in 1 -well of a 6-well plate. Total RNA was extracted from infected cells using TRIzol (Invitrogen) as per manufacturer's instructions. RNA was DNase treated using the Ambion DNase-free kit, as per the manufacturer's instructions, and total RNA $(1 \mu \mathrm{g})$ from each fraction was reverse transcribed with SuperScript ${ }^{\mathrm{TM}}$ II (Invitrogen), as per the manufacturer's instructions, using oligo(dT) primers (Promega). cDNA (10 ng) was used as template in SensiMixPlus SYBR qPCR reactions (Quantace), as per manufacturer's instructions, using a Rotor-Gene Q 5plex HRM Platform (Qiagen), with a standard 3-step melt program $\left(95^{\circ} \mathrm{C}\right.$ for $15 \mathrm{sec}, 60^{\circ} \mathrm{C}$ for $30 \mathrm{sec}, 72^{\circ} \mathrm{C}$ for $20 \mathrm{sec}$ ). With GAPDH as internal control mRNA, quantitative analysis was performed using the comparative $\mathrm{C}_{\mathrm{T}}$ method as previously described (31).

Flow cytometry. Apoptosis was induced in cells with soluble TRAIL protein (TRIMOL) at a concentration of $0.5 \mu \mathrm{g} / \mathrm{ml}$ in 10\% DMEM. The Jak inhibitor AG490 was used to enhance TRAIL-mediated apoptosis in certain cells, at a concentration of $100 \mathrm{mM}$. For cell analysis, treated cells were trypsinised and resuspended in their original medium. Cells $\left(5 \times 10^{6}\right)$ were then washed twice with annexin $\mathrm{V}$ labelling buffer $(10 \mathrm{mM}$ HEPES (pH 7.4), $140 \mathrm{mM} \mathrm{NaCl}, 2.5 \mathrm{mM} \mathrm{CaCl}_{2}$ ), resuspended in $50 \mu 1$ labelling buffer and stained with annexin V-Cy5 (BD Biosciences) and propidium iodide (Sigma) according to the 
manufacturer's instructions. Fluorescence was measured using the FL4 (annexin V-Cy5) and FL3 (PI) channels on a BectonDickinson FACSCalibur flow cytometer, and the CellQuest software was used to estimate the proportion of cells in the early and late stages of apoptosis.

Determination of caspase activity. Caspase-Glo ${ }^{\circledR} 8$ and Caspase-Glo 3/7 assays were obtained from Promega, and experiments were performed in triplicate. Cells were seeded into white-walled 96-well plates, and infected with HVS $\Delta 71-73$ TRAIL, or treated with sTRAIL in a total volume of $50 \mu 1$ media (media-only samples were used as background controls). After the appropriate incubation time (6-48 h), $50 \mu \mathrm{l}$ Caspase-Glo reagent was added to each well and incubated at room temperature for $1 \mathrm{~h}$ according to the manufacturer's instructions. Luminescence was then measured using a BMG Labtech Fluostar plate reader. The averages of the readings from the media-only wells were subtracted from the data to decrease background signal.

\section{Results}

Construction of HVS $\Delta 71-73$ TRAIL. The recombinant HVS used has been modified by the insertion of an F-factor-based bacterial artificial chromosome (BAC) element into ORF15 (29). The BAC element enables the HVS genome to be maintained in dividing populations of $E$. coli. It also contains a chloramphenicol resistance gene for selection, and introduces a unique I-PpoI restriction site into the episome so that exogenous DNA can be inserted by standard excision/ligation techniques. Initially, the TRAIL expression cassette used was cloned into this HVS BAC. However this construct, HVS-GFP-TRAIL, was unable to induce apoptosis due to the expression of a viral FLICE inhibitory protein (FLIP), expressed from ORF71 (32). Therefore the HVS BAC utilised in these studies was reconstructed using a virus in which the latently expressed genes, ORFs 71-73 have been removed. We have previously described the construction and analysis of the HVS $\Delta 71-73$ virus (9).

HVS $\Delta 71-73$ TRAIL was produced in a two-step process using traditional cloning techniques (Fig. 1a). The TRAIL expression cassette consists of the human TRAIL full length gene controlled by the tumour specific human $\alpha$-survivin promoter. This cassette was inserted into a shuttle vector prior to being incorporated into the I-PpoI site of HVS $\Delta 71-73$. Positive clones were identified by chloramphenicol/kanamycin screening, and further analysed by I-PpoI restriction digest and pulse field gel electrophesis (Fig. 1b). The 2.7-kb TRAIL cassette has clearly been excised from the $\sim 160 \mathrm{~kb}$ HVS genome, indicating successful insertion (this was also confirmed by sequencing analysis - data not shown). To attain stocks of infectious viral particles, the HVS $\Delta 71-73$ TRAIL DNA was transfected into permissive OMK cells, and viral particles were harvested in the media once the cell sheet was completely lysed. Progress of the infection could be observed due to expression of the dsRed marker gene.

TRAIL is expressed from HVS $\triangle 71-73$ TRAIL in colorectal SW480 cells. To confirm expression of TRAIL in HVS $\Delta 71-73$ TRAIL-infected cells, TRAIL mRNA levels in infected SW480 cells was assessed using qRT-PCR. RNA was extracted from SW480 infected cells, 24 h post-infection. As a positive control,

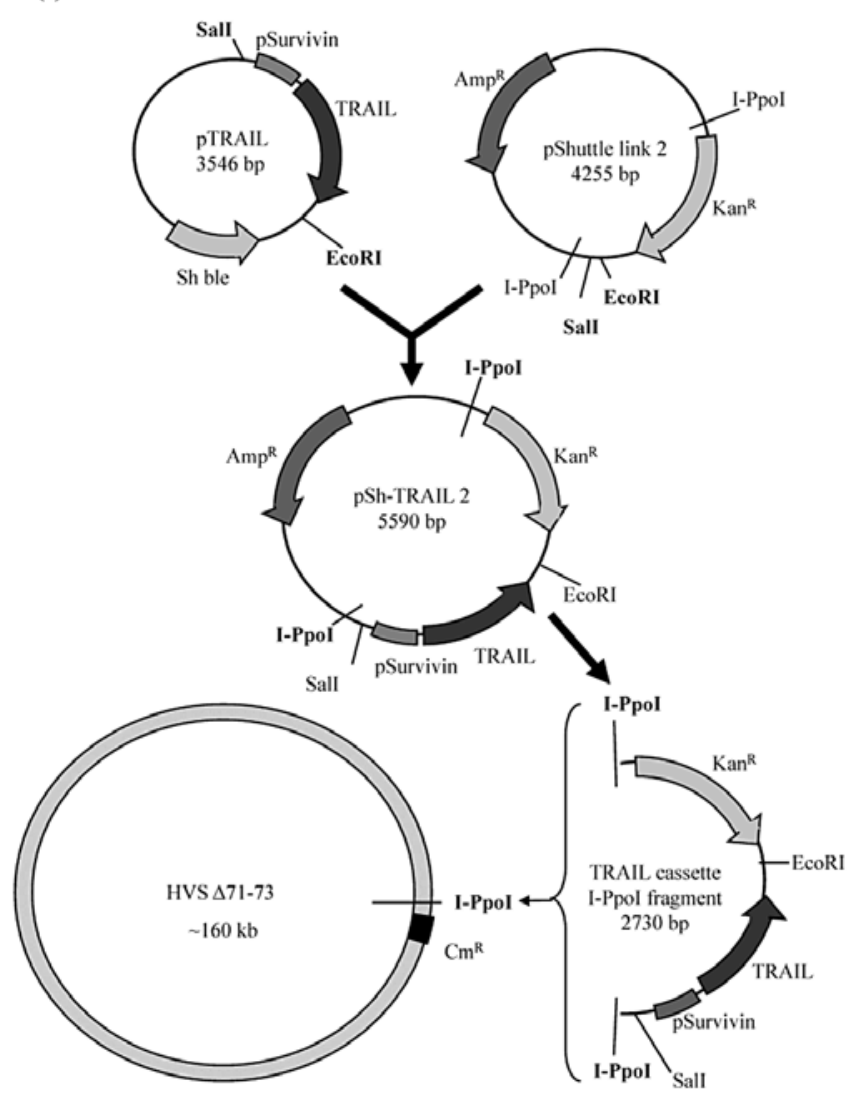

(b)

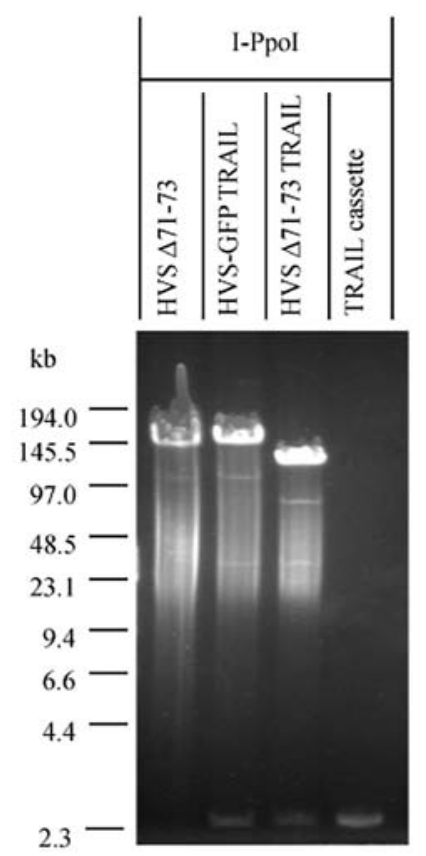

Figure 1. Construction of HVS $\Delta 71-73$ TRAIL. (a) Schematic of cloning procedure. pShuttle link 2 and pTRAIL fragments were ligated after a SalI/ EcoRI digest. The resulting plasmid, pSh-TRAIL 2, and the HVS-BAC were digested with I-PpoI and subsequently ligated. Transformants were selected on Kan and chloramphenicol $(\mathrm{Cm})$ plates, and insertion was checked by DNA sequencing. (b) Diagnostic I-PpoI digest of HVS $\Delta 71-73$ TRAIL confirms the presence of the $2.7 \mathrm{~kb}$ insert corresponding to the TRAIl expression cassette (confirmed by sequencing). Digested DNA was run on a pulse field electrophoresis gel.

cells transfected with the original pTRAIL plasmid, from which the TRAIL cassette was obtained, were also used. pEGFP-c1 


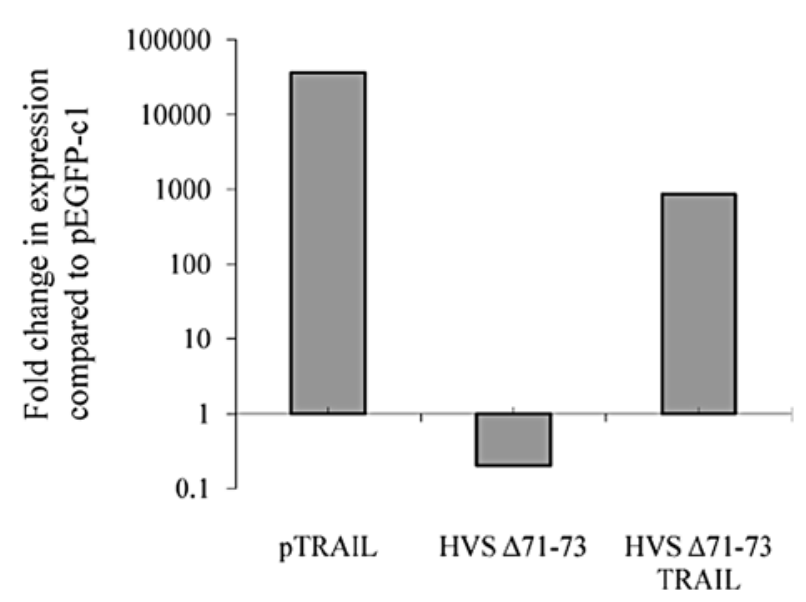

Figure 2. TRAIL expression from HVS $\Delta 71-73$ TRAIL as measured by RT-qPCR in SW480 cells. Cells were either transfected with $1 \mu \mathrm{g}$ pEGFP-c1 or pTRAIL, or infected with HVS $\Delta 71-73$, or HVS $\Delta 71-73$ TRAIL. Twenty-four hours later RNA was extracted and RT qPCR was performed. The comparative $\mathrm{C}_{\mathrm{T}}$ method was used to establish the fold change in TRAIL expression compared to the pEGFP-c1 transfected samples. pTRAIL transfected cells displayed the highest TRAIL mRNA levels, however HVS $\triangle 71-73$ TRAIL infection also caused a substantial increase. The difference in expression may be due to the low copy number of the viral genome or differing transfection efficiencies.

transfected cells and HVS $\Delta 71-73$ infected cells were negative controls. Fig. 2 shows the fold change in TRAIL expression at the mRNA level, compared to the pEGFP-cl transfected sample, indicating expression of the transgene from HVS $\Delta 71-73$ TRAIL.

HVS A71-73 TRAIL induces apoptosis in colorectal cancer $S W 480$ cells. In order to assess the effect of HVS $\Delta 71-73$ TRAIL infection in cancerous cells, apoptosis induction was measured with a flow cytometry assay using cells labelled with annexin $\mathrm{V}$ and propidium iodide. Images of the SW480 cells were also taken to observe any gross changes in morphology. Measurements were taken at 24,48 and $72 \mathrm{~h}$ time-points with untreated SW480 cells, cells treated with $0.5 \mu \mathrm{g} / \mathrm{ml}$ soluble TRAIL protein (sTRAIL), and cells infected with HVS $\Delta 71-73$ or HVS $\Delta 71-73$ TRAIL, respectively. As predicted, sTRAIL induced apoptosis in the majority of cells by the 24-h time-point. The HVS $\Delta 71-73$ virus did not increase cell death over the 72-h time course. In contrast, the HVS $\Delta 71-73$ TRAIL virus had a dramatic effect on cell viability. By $72 \mathrm{~h}$ post-infection, virtually all of the cells were floating in the wells and had clearly undergone cell death. This observation is supported by the flow cytometry data in Fig. $3 b$. At the $48 \mathrm{~h}$ time-point $>60 \%$ of the population were classed as undergoing cell death, and this increased to almost $100 \%$ at $72 \mathrm{~h}$.

Some of the dot plots obtained in the flow cytometry analysis are displayed in Fig. $3 \mathrm{c}$. The $\Delta 71-73$ TRAIL infected samples can be clearly seen to shift from the main healthy population, being double-negative for the annexin V and PI stains at $24 \mathrm{~h}$, to a significant proportion staining positive for annexin $\mathrm{V}$ at $48 \mathrm{~h}$. By $72 \mathrm{~h}$ the cells are either double positive or PI-positive. This shift follows the progressive stages of apoptosis. Soluble TRAIL protein acted faster at inducing apoptosis than the $\Delta 71-73$ TRAIL, and this lag may be due to a delay in transgene expression. However the $\Delta 71-73$ TRAIL appears more efficient than sTRAIL at inducing cell death by $72 \mathrm{~h}$, and this may be due to continuous TRAIL production.
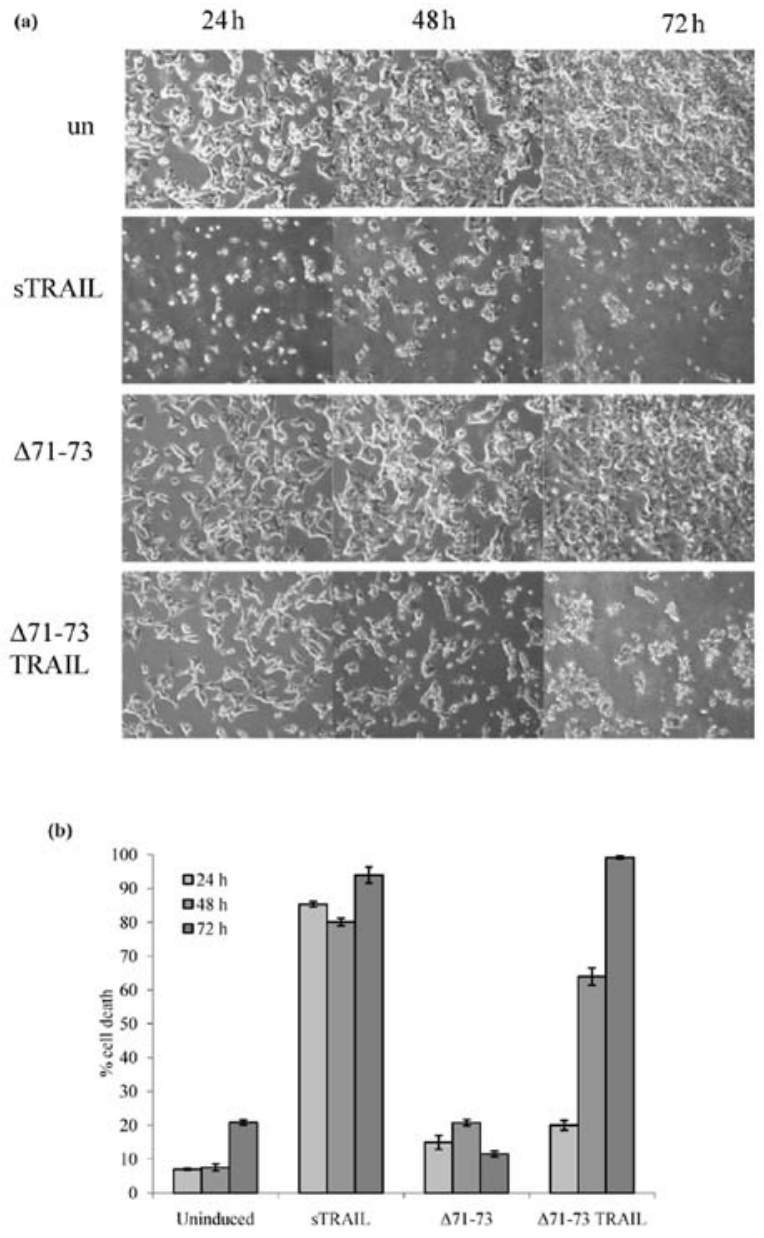

(c)

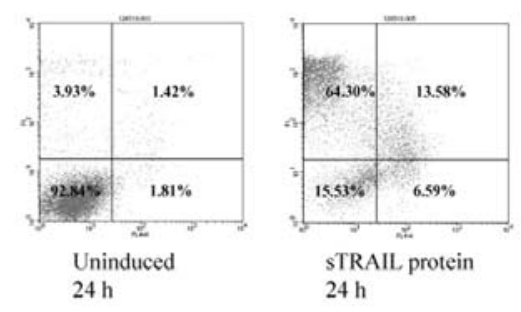

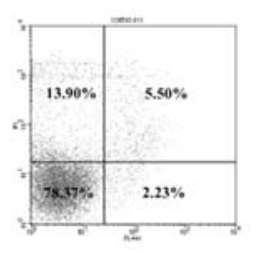

HVS $\triangle 71-73$ TRAIL $24 \mathrm{~h}$

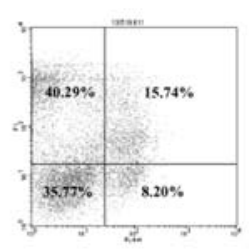

HVS $\triangle 71-73$ TRAIL $48 \mathrm{~h}$

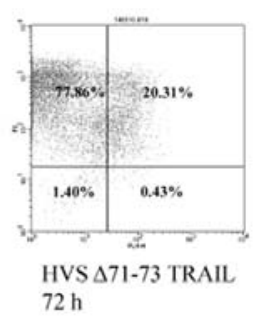

$72 \mathrm{~h}$
Figure 3. HVS $471-73$ TRAIL induces cell death in SW480 colorectal cancer cells. (a) Microscope images show that untreated cells and HVS $\Delta 71-73$ infected cells continue to grow over the 72-h experiment. Cells treated with soluble TRAIL (sTRAIL) have undergone apoptosis by $24 \mathrm{~h}$, and the HVS $\Delta 71-73$ TRAIL infected cells shrink and become rounded at $48 \mathrm{~h}$ post-infection, and have all lifted from the plate surface at $72 \mathrm{~h}$ post-infection. (b) Flow cytometry analysis using annexin V/PI staining clearly shows the increase in apoptotic cells upon infection of HVS $\Delta 71-73$ TRAIL. The percentage cell death shown is all cells not in the lower left quadrant of the dot plots obtained (i.e., not live annexin V-negative, PI-negative). Cell death in HVS $\Delta 71-73$ TRAIL infected cells increases from $20 \%$ to almost $100 \%$ at $72 \mathrm{~h}$ ( $\mathrm{n}=3$, error bars, SD). (c) HVS $\Delta 71-73$ TRAIL infected cells undergo apoptosis. The flow cytometry dot plots show increasing numbers of annexin V-positive cells (x-axis) throughout the time course, indicating that cells infected with HVS $\Delta 71-73$ TRAIL are early apoptotic at $48 \mathrm{~h}$ and late apoptotic by $72 \mathrm{~h}$. 
(a)

$6 \mathrm{~h}$

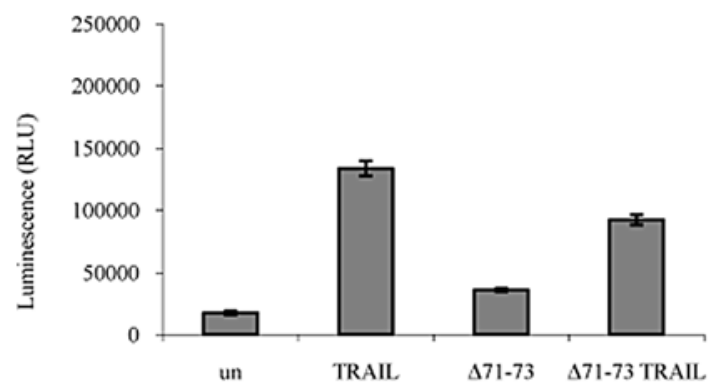

$24 \mathrm{~h}$

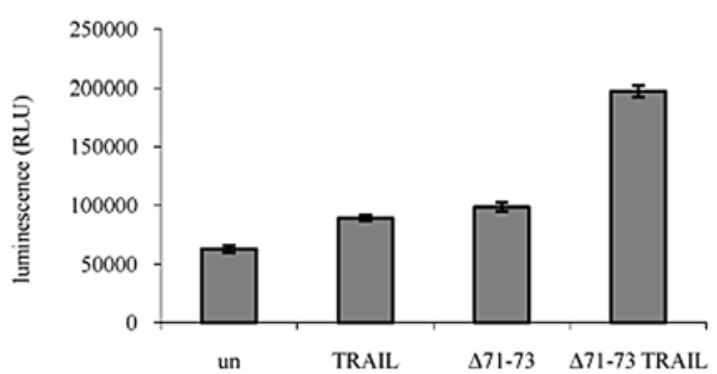

$48 \mathrm{~h}$

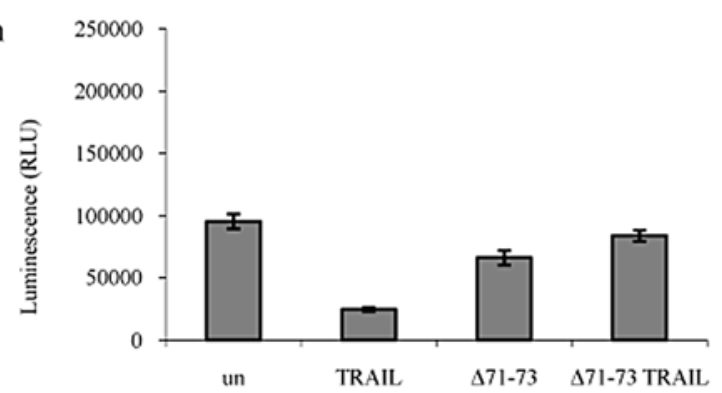

(b)

$6 \mathrm{~h}$

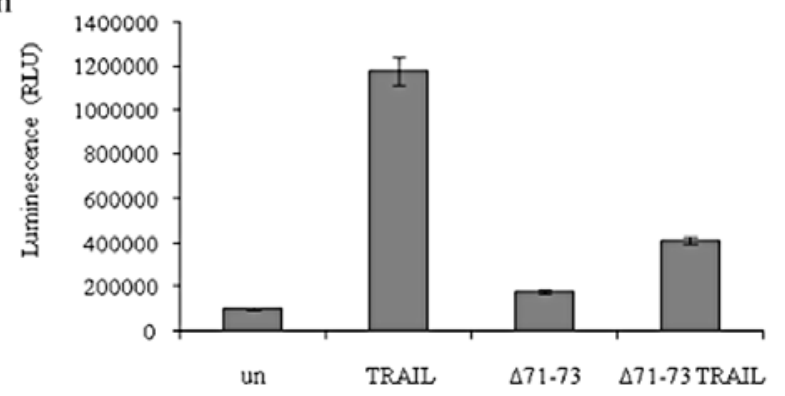

$24 \mathrm{~h}$
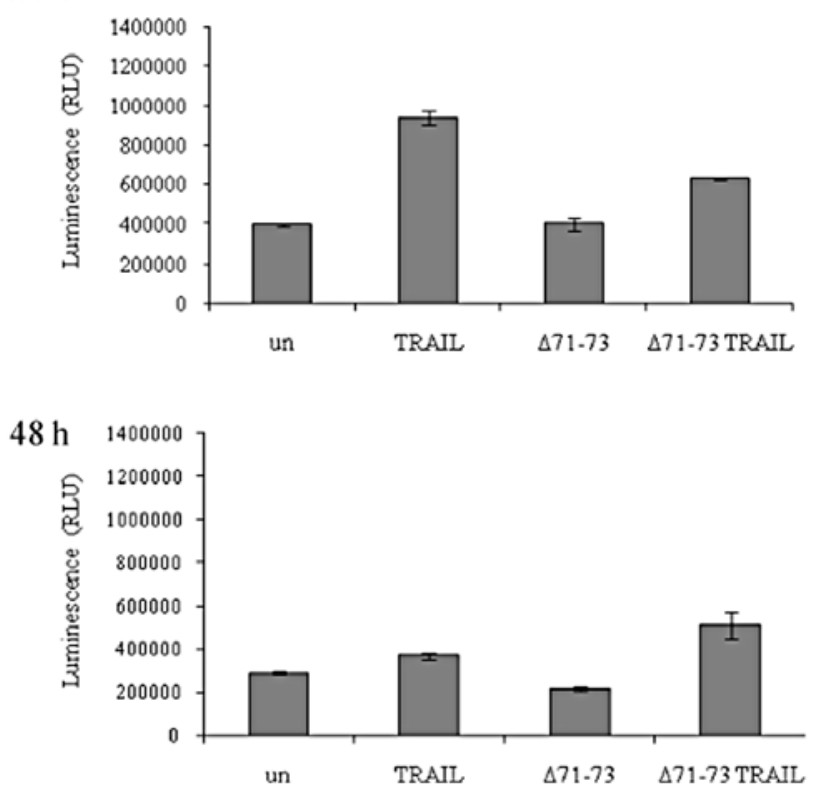

Figure 4. HVS $\Delta 71-73$ TRAIL upregulates caspase-8 and caspase-3/7 activity in SW480 cells. Cells were incubated with $0.5 \mu \mathrm{g} / \mathrm{ml}$ soluble TRAIL protein, HVS $\Delta 71-73$, and HVS $\Delta 71-73$ TRAIL for 6,24 and $48 \mathrm{~h}$, after which caspase-8 or -3/7 activity was assessed using the Caspase-Glo Assay System (Promega). Luciferase activity was measured using a BMG Labtech Fluostar plate reader ( $\mathrm{n}=3$, error bars, SD). (a) Caspase- 8 activity peaked at $6 \mathrm{~h}$ in sTRAIL treated cells whereas activity in the HVS $\Delta 71-73$ TRAIL infected cells was highest at $24 \mathrm{~h}$. (b) Similarly, caspases-3 and -7 were most active $6 \mathrm{~h}$ after treatment with sTRAIL, but caspase activity was more stable over the 72-h period in HVS $\Delta 71-73$ TRAIL infected cells.

HVS 471-73 TRAIL infection causes increased caspase activity in SW480 cells. To further investigate the mechanism of action of HVS $\Delta 71-73$ TRAIL, caspase activity was measured in infected SW480 cells using a Caspase-Glo Assay System (Promega). This assay measures caspase activity through the cleavage of a caspase-specific substrate which is in turn oxidised by luciferase, producing measurable light emission. HVS $\Delta 71-73$ TRAIL infection was compared with untreated, HVS $\Delta 71-73$ infected and sTRAIL treated cells at $6 \mathrm{~h}, 24 \mathrm{~h}$, and $48 \mathrm{~h}$ post infection/ induction.

Fig. 4a shows the results for activity of the initial caspase in the extrinsic apoptosis pathway, caspase- 8 . After $6 \mathrm{~h}$ caspase- 8 activity is dramatically increased in both the sTRAIL and HVS $\Delta 71-73$ TRAIL samples compared to the untreated sample. The cells treated with STRAIL show a higher increase in activity at this time-point. However, at $24 \mathrm{~h}$, the HVS $\Delta 71-73$ TRAIL infected cells show the most caspase- 8 activity. By $48 \mathrm{~h}$, this activity is diminished in both of the samples containing TRAIL. The caspase- 8 activity in the HVS $\Delta 71-73$ infected cells remains relatively low throughout the time course. These data indicate that sTRAIL strongly induces the apoptotic cascade via the extrinsic pathway, but this signal is transient and activity is similar to the uninduced sample by $24 \mathrm{~h}$. In contrast, caspase- 8 activity increases gradually in the HVS $\Delta 71-73$ TRAIL samples, and is sustained for a longer period in infected cells.

The activity of executioner caspases-3 and -7was also assessed. The activity of these caspases, as measured by luminescence, is shown in Fig. 4b, using the same 6, 24 and $48 \mathrm{~h}$ time-points. Cells treated with sTRAIL have a large increase in caspase-3/7 activity at $6 \mathrm{~h}$, which slowly returns to the same level as the untreated cells by $48 \mathrm{~h}$. Caspase-3/7 activity in the HVS $\Delta 71-73$ infected cells remains at the same level as untreated cells for all 3 measurements. In the HVS $\Delta 71-73$ TRAIL infected cells, activity is slightly increased at $6 \mathrm{~h}$, and remains at this level throughout the 3 time-points. The data are consistent with the results of the caspase- 8 activity assay and indicates that caspase cleavage in sTRAIL-mediated apoptosis occurs within $6 \mathrm{~h}$ of death receptor binding. There is a delay in caspase activity in HVS $\Delta 71-73$ TRAIL infected cells, compared to STRAIL treated cells. Also, both caspase- 8 and caspase- $3 / 7$ activity is more sustained in infected cells. 
HVS A71-73 TRAIL causes cell death in several human cancer cell lines. As the HVS $\Delta 71-73$ TRAIL virus was able to successfully induce apoptosis in the colorectal cancer cell line SW480, the effect of the virus on other human cancer cell lines was assessed. HVS $\Delta 71-73$ TRAIL, at m.o.i 1, was used to infect 9 cell lines including cells derived from liver, lung, pancreatic, prostate and cervical cancer. Forty-eight hours post-infection, flow cytometry was used to assess the percentage of cells undergoing cell death. Cells were also challenged with STRAIL to identify cell lines which are TRAIL resistant, and HVS $\Delta 71-73$ to account for any virus toxicity. Fig. 5a displays the results for all 9 cell lines.

The colorectal SW480 cell line was the most sensitive to $0.5 \mu \mathrm{g} / \mathrm{ml}$ sTRAIL, and was also very sensitive to HVS $\Delta 71-73$ TRAIL, as expected. The hepatocellular carcinoma cell lines Huh7, Huh7.5 and HepG2 were fairly resistant to sTRAIL. However almost $50 \%$ of the A549 lung carcinoma cells infected with HVS $\Delta 71-73$ TRAIL underwent cell death, which is somewhat surprising as neither sTRAIL alone nor HVS $\Delta 71-73$ induced a similar effect. It is possible that HVS infection creates an environment in the cell that allows apoptosis to occur more easily, possibly due to sustained expression of TRAIL.

Another interesting result was observed with the MiaPaCa2 pancreatic cancer cells. These cells were sensitive to sTRAIL, as $65 \%$ underwent cell death after $48 \mathrm{~h}$, but the same level of cell death was observed with HVS $\Delta 71-73$ infected cells. This toxicity upon HVS infection has been previously observed in both MiaPaCa2 and Panc1 pancreatic cancer cells (33). There appears to be no additive effect between the virus and TRAIL, as HVS $\Delta 71-73$ TRAIL induces the same level of cell death. Moreover, upon analysis of the flow cytometry dot plots obtained under these different conditions (Fig. 5b), it appears that the majority of cells undergoing cell death in the HVS $\Delta 71-73$ TRAIL infected sample are annexin V-positive, and the general staining pattern is similar to the sTRAIL treated cells. This indicates that these cells are apoptotic rather than necrotic. In contrast only $12 \%$ of the HVS $\Delta 71-73$ infected cells are gated as annexin V-positive, which is lower than even the untreated sample. The majority of cells are PI-positive, which is more characteristic of a necrotic form of cell death. Apoptosis, as observed with the HVS $\Delta 71-73$ TRAIL infected cells, may be advantageous for gene therapy purposes as it is less likely to stimulate an inflammatory response.

A similar cytopathic effect is seen in the prostate cancer cell line PC3 in Fig. 5a, but the toxicity is far less pronounced. Cells infected with HVS $\Delta 71-73$ underwent $30 \%$ cell death, and displayed similar levels of cell death with both sTRAIL and HVS $\Delta 71-73$ TRAIL, although this is only a $10-15 \%$ increase on the uninduced samples. The Ewing's sarcoma cell line TC32 was fairly resistant to TRAIL but infection with HVS $\Delta 71-73$ TRAIL did increase cell death by $\sim 10 \%$. Finally, the cervical cancer cell line CaSki was slightly responsive to sTRAIL, but did not show a significant increase in cell death when infected with HVS $\Delta 71-73$ TRAIL.

HVS 471-73 disrupts multicellular spheroid cultures in combination with the Jak inhibitor AG490. Previous studies have shown that HVS is able to efficiently infect and penetrate multicellular spheroid cultures similar to tumours in vitro (12), providing a far more realistic model of human cancer than cell monolayers. Following this observation, we sought to demonstrate the efficacy (a)

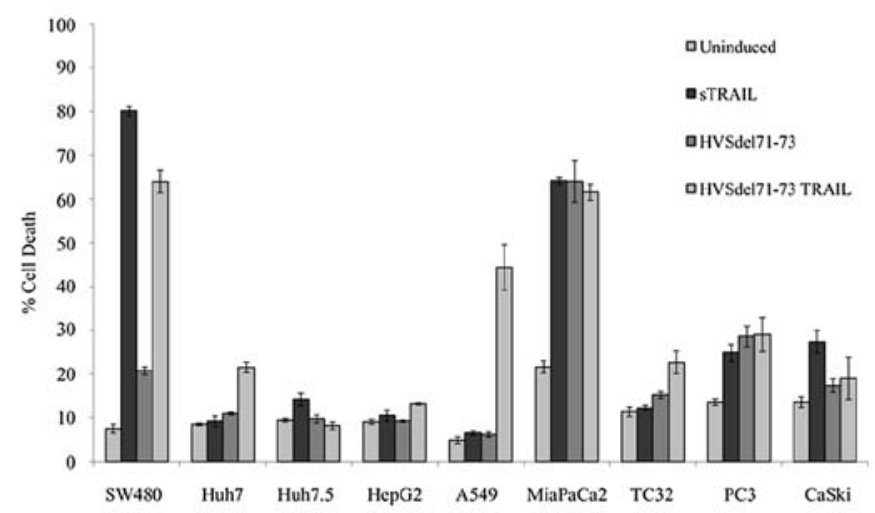

(b)
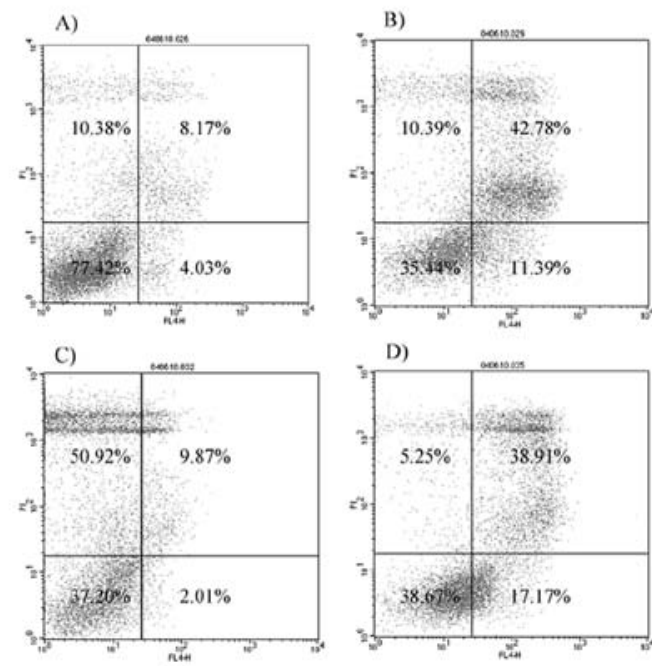

Figure 5. HVS $\Delta 71-73$ TRAIL induces cell death in several human cancer cell lines. (a) Nine cell lines derived from different human cancers were treated with either $0.5 \mu \mathrm{g} / \mathrm{ml}$ sTRAIL, or infected with HVS $\Delta 71-73$, or HVS $\Delta 71-73$ TRAIL. After $48 \mathrm{~h}$ the percentage of cells undergoing cell death was measured using an annexin V/PI flow cytometry assay ( $n=3$, error bars, SD). (b) MiaPaCa2 cells exhibit cytopathic effects when infected with HVS $\Delta 71-73$, but appear apoptotic when infected with HVS $\Delta 71-73$ TRAIL. Dot plots of either uninduced cells (A), or cells treated with $0.5 \mu \mathrm{g} / \mathrm{ml}$ sTRAIL (B), HVS $\Delta 71-73(\mathrm{C})$, or HVS $\Delta 71-73$ TRAIL (D) were incubated for $48 \mathrm{~h}$ prior to flow cytometry analysis with annexin V/PI staining. The sTRAIL treated cells have a population of annexin V-positive cells in the bottom of the upper right quadrant, and a second population higher up. This is also seen in the HVS $\Delta 71-73$ TRAIL infected cells, indicating apoptosis. In contrast, the majority of the HVS $\Delta 71-73$ sample is annexin V-negative, and $50 \%$ PI-positive, suggesting necrosis.

of HVS $\Delta 71-73$ TRAIL in shrinking or degrading these types of cultures. The melanoma cell line Mel888 was used to form these structures. Mel888 cells are not as sensitive to HVS $\Delta 71-73$ TRAIL infection as the SW480 cell line, however it has been shown that TRAIL sensitivity can be improved by down-regulation of pro-inflammatory and anti-apoptotic protein activity. Several studies have highlighted the anti-apoptotic effects of the signalling molecule STAT3. This protein is constitutively active in many cancer cell lines, upregulating expression of several IAPs (34-36). Inhibition of STAT3 activation by the Jak inhibitor AG490 has been shown to increase TRAIL sensitivity (37), therefore we investigated whether this JAK inhibitor could sensitise the cells to HVS $471-73$ TRAIL. Mel888 cells grown as an adherent cell monolayer were incubated with $50 \mu \mathrm{M}$ AG490 and HVS $\Delta 71-73$ TRAIL at m.o.i 1 for $48 \mathrm{~h}$, and then 


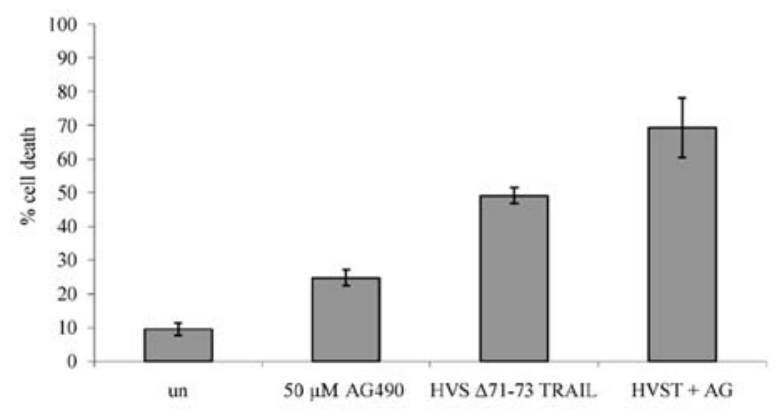

(b)

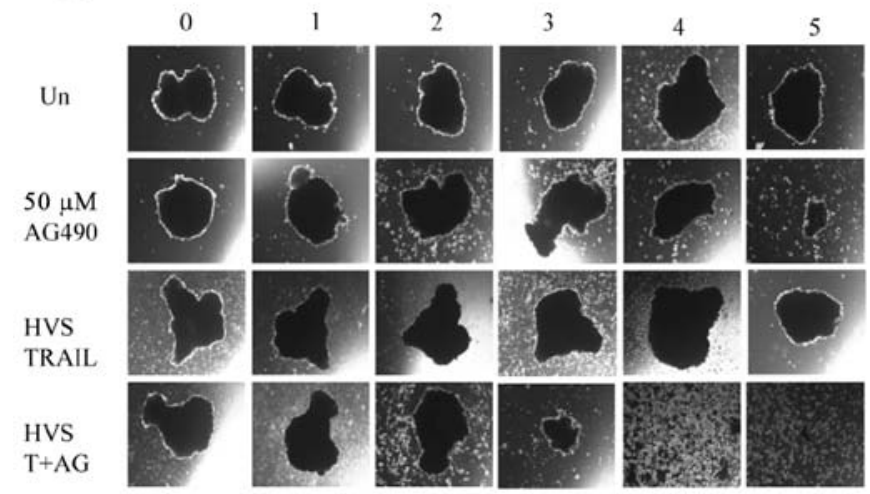

Figure 6. HVS $\Delta 71-73$ TRAIL disperses spheroid cultures of the Mel888 cell line in combination with Jak inhibitor AG490. (a) Annexin V/PI apoptosis assay shows that, although HVS $\Delta 71-73$ TRAIL causes approximately $50 \%$ cell death after 48-h incubation, this is increased to approximately $70 \%$ in the presence of $50 \mu \mathrm{M}$ AG490. (b) Microscope images of spheroid cultures treated with $50 \mu \mathrm{M}$ AG490, HVS $\Delta 71-73$ TRAIL at an m.o.i of 2, or combined treatment. HVS $\Delta 71-73$ TRAIL infection alone appears to have no effect on spheroid structure, but combined treatment efficiently disperses spheroids 4 days post-infection. AG490 treatment also has an effect on spheroid size, but to a lesser extent than combined treatment.

analysed by flow cytometry (Fig. 6a). A combined effect could be seen between the Jak inhibitor and the virus, resulting in $\sim 70 \%$ cell death.

Following this, we investigated whether HVS $\Delta 71-73$ TRAIL had any effect on spheroids grown from Mel888 cells. Spheroids were grown for 2 weeks in a T75 prior to infection. Spheroids were then transferred to a 6 -well plate (one spheroid per well) and either used as a control, infected with HVS $\Delta 71-73$ TRAIL, treated with $50 \mu \mathrm{M}$ AG490, or treated with both agents. Microscope images were captured every $24 \mathrm{~h}$ to observe changes in spheroid structure. Fig. $6 \mathrm{~b}$ is representative of the results seen. Untreated spheroids maintained similar dimensions over the 5-day time course. Significantly, the HVS $\Delta 71-73$ TRAIL infected spheroids also did not exhibit any change in size or shape. The AG490 treated spheroids did start to lose some structural integrity by 3 days post-treatment, and broke apart into smaller structures by day 5 . However, dual treated spheroids were visibly reduced in size by day 3 and had completely disintegrated by day 4. This differed from the AG490 treated samples which broke into smaller spheroids, whereas dual treated samples seemed to fragment into individual cells.

\section{Discussion}

TRAIL has been regarded as a 'holy grail' of cancer therapy due to its highly selective action (38). As such, several therapies with this protein have undergone clinical trials $(39,40)$. We have produced a novel viral vector based on Herpesvirus saimiri, which allows continuous albeit short-term expression of TRAIL. This vector can infect and initiate apoptosis in several cancer cell lines, and shows promise for treatment in TRAIL-sensitive cancers.

HVS $\Delta 71-73$ TRAIL infection of SW480 cells induced a pronounced apoptotic effect. Almost $100 \%$ of the colorectal cancer cells had undergone cell death by $72 \mathrm{~h}$ post-infection, which was comparable to sTRAIL treatment at $24 \mathrm{~h}$ post-induction. The lag compared to the effects of the sTRAIL protein preparation is probably due to expression of the transgene taking a greater time to reach sufficient levels to induce apoptosis. Caspase activity assays showed that both caspase- 8 and caspases-3/7 were activated by HVS $\Delta 71-73$ TRAIL infection. Again, caspase activation occurs at a later time-point compared with SW480 cells challenged with sTRAIL, but activation appears to be more sustained with HVS $\Delta 71-73$ TRAIL infection. This supports a model that, upon infection, full length TRAIL is continuously expressed from the $\alpha$-survivin promoter, and is translocated to the plasma membrane where the membrane-bound protein interacts with death receptors on neighbouring cells. It is also possible that cleaved soluble TRAIL mediates a more far reaching apoptotic effect, however this was not investigated. A similar study investigating the bystander effect of an adenovirus-based vector expressing full length human TRAIL in liver cancer cells found that apoptosis induction was mainly cell-mediated, rather than being caused by a secreted soluble component, suggesting that the majority of expressed TRAIL was membrane bound (41). It is likely that a similar surface expression of TRAIL is occurring with this HVS $\Delta 71-73$ TRAIL, however this could be determined by transferring the culture medium of infected cells to fresh SW480 cells and measuring apoptosis induction.

As HVS $471-73$ TRAIL was able to elicit such a dramatic effect in SW480 cells, this virus was then used to infect a range of human cancer cell lines. Several of these were sensitive to this treatment, including the pancreatic MiaPaCa2 line and the A549 lung carcinoma line. From these observations, and as expected, it seems that cells that are sensitive to sTRAIL are also sensitive to HVS $\Delta 71-73$ TRAIL. However, as in the case of the A549 lung carcinoma cells, the virus can induce apoptosis when cells are not especially susceptible to TRAIL treatment, and this may be due to cell signalling pathways that are activated upon infection, or due to sustained TRAIL expression.

In addition, some cytopathic effects of HVS infection were seen in MiaPaCa2, and to a lesser extent in prostate cell line PC3. It has previously been reported that HVS causes cytopathic effects in pancreatic cancer cells (33). The annexin V/PI flow cytometry assay used in our system suggests that the process of cell death is likely to be necrosis, rather than apoptosis. Stevenson et al (33), suggested that, although the likely explanation for this observation is lytic virus production, it is also possible that a viral gene product is responsible for the cell death. This second theory seems to agree more with the flow cytometry data obtained with HVS $\Delta 71-73$ TRAIL, as $48 \mathrm{~h}$ would be too short for over $65 \%$ of the cell sheet to lyse due to virus production. 
To further investigate the activity of HVS $\Delta 71-73$ TRAIL, we infected melanoma spheroid cultures. Although HVS $\Delta 71-73$ alone did not reduce spheroid size, co-treatment with the Jak inhibitor AG490 caused the cellular structures to disperse. The synergistic action of these agents is thought to be due to the inhibition of downstream STAT3 signalling, which is implicated in the progression of many cancers, including melanoma (42). STAT3 upregulates a host of anti-apoptotic proteins such as Bcl-xL, therefore blocking this signal can sensitise cells to apoptosis induction.

Initial attempts to develop a TRAIL expressing HVS vector were unsuccessful at inducing apoptosis. This inhibition was found to be caused by the viral gene ORF71, which encodes a FLICE (caspase-8) inhibitor protein (v-FLIP) (32). HVS v-FLIP contains two death effector domains (DEDs) which are capable of binding FADD, thereby forming a DISC-like complex with associated death receptors $(24,32)$ This interaction competitively inhibits the recruitment of caspase-8, preventing its cleavage and activation, and effectively stopping the apoptotic signal.

To address the problem of apoptosis inhibition, the polycistronic region encoding ORFs 71-73 was removed from HVS. One consequence of disrupting this region is the loss of expression of the episomal maintenance factor, ORF73. However, as this therapy is designed to eradicate the target cells within a relatively short time scale, this should not adversely affect the efficacy of the vector. As seen with HVS $\Delta 71-73$ TRAIL virus, sufficient TRAIL was expressed from infected cells within $72 \mathrm{~h}$ to cause a considerable apoptotic effect in SW480 cells.

To resolve the problems that can arise due to viral protein expression, ideally a 'gutless' amplicon HVS vector would be used. The present amplicon system developed by Macnab et al (15), still contains ORF71 so is unsuitable for use with a TRAIL transgene, but with further development this would be a valuable platform to build gene therapy constructs.

One of the major advantages of TRAIL as an anti-cancer agent is that its activity is specific to cancer cells. However, to further enhance the safety of HVS $\Delta 71-73$ TRAIL, we also utilised transcriptional targeting, whereby TRAIL was placed under the control of a tumour-specific promoter. The promoter from $\alpha$-survivin was chosen to drive the transgene as survivin expression is upregulated in the majority of cancers while being undetectable in terminally differentiated adult tissues (43). Survivin is an IAP which plays a crucial role in cell cycle regulation and cell survival $(43,44)$. This protein can block apoptosis through direct binding of caspases and through interaction with Smac/DIABLO $(45,46)$. The use of this promoter gives an extra level of fidelity to the gene therapy as the transgene is expressed only in cells which also express survivin.

In conclusion, herein we have shown for the first time that an HVS $\Delta 71-73$ TRAIL vector shows great promise as an anti-cancer agent, either alone or in combination with compounds that enhance apoptosis, such as the Jak inhibitor AG490. Further characterisation of the molecular effects of HVS $\Delta 71-73$ TRAIL infection, both in vitro and in vivo, is required to assess bystander effects, safety, and efficacy in healthy and neoplastic cells. However, we have provided the first proof of principle that HVS-based vectors can deliver a transgene which produces a therapeutic effect for effective cancer treatment.

\section{Acknowledgements}

We thank Gareth Howell for guidance regarding the Flow Cytometry experiments. This work was supported by in parts by a BBSRC DTG studentship and a studentship funded by the University of Leeds Interdisiplinary Institute in Bionanoscience to A.W. A.W. is a recipient of a BBSRC research development fellowship.

\section{References}

1. Falk LA, Wolfe LG and Deinhardt F: Isolation of Herpesvirus saimiri from blood of squirrel monkeys (Saimiri sciureus). J Natl Cancer Inst 48: 1499-1505, 1972.

2. Griffiths RA, Boyne JR and Whitehouse A: Herpesvirus saimiribased gene delivery vectors. Curr Gene Ther 6: 1-15, 2006.

3. Calderwood MA, White RE and Whitehouse A: Development of herpesvirus-based episomally maintained gene delivery vectors. Expert Opin Biol Ther 4: 493-505, 2004.

4. Desrosiers RC, Kamine J, Bakker A, et al: Synthesis of bovine growth hormone in primates by using a herpesvirus vector. Mol Cell Biol 5: 2796-2803, 1985.

5. Whitehouse A: Herpesvirus saimiri: a potential gene delivery vector (Review). Int J Mol Med 11: 139-148, 2003.

6. Simmer B, Alt M, Buckreus I, et al: Persistence of selectable Herpesvirus saimiri in various human haematopoietic and epithelial cell lines. J Gen Virol 72: 1953-1958, 1991

7. Grassmann R and Fleckenstein B: Selectable recombinant herpesvirus saimiri is capable of persisting in a human T-cell line. J Virol 63: 1818-1821, 1989.

8. Stevenson AJ, Clarke D, Meredith DM, Kinsey SE, Whitehouse A and Bonifer C: Herpesvirus saimiri-based gene delivery vectors maintain heterologous expression throughout mouse embryonic stem cell differentiation in vitro. Gene Ther 7: 464-471, 2000.

9. Calderwood M, White RE, Griffiths RA and Whitehouse A: Open reading frame 73 is required for herpesvirus saimiri A11-S4 episomal persistence. J Gen Virol 86: 2703-2708, 2005.

10. Griffiths R and Whitehouse A: Herpesvirus saimiri episomal persistence is maintained via an interaction between open reading frame 73 and the cellular chromosome associated protein MeCP2. J Virol 81: 4021-4032, 2007.

11. Calderwood MA, Hall KT, Matthews DA and Whitehouse A: The herpesvirus saimiri ORF73 gene product interacts with host-cell mitotic chromosomes and self-associates via its $\mathrm{C}$ terminus. J Gen Virol 85: 147-153, 2004.

12. Smith PG, Burchill SA, Brooke D, Coletta PL and Whitehouse A: Efficient infection and persistence of a herpesvirus saimiri-based gene delivery vector into human tumor xenografts and multicellular spheroid cultures. Cancer Gene Ther 12: 248, 2004.

13. Smith PG, Coletta PL, Markham AF and Whitehouse A: In vivo episomal maintenance of a herpesvirus saimiri-based gene delivery vector. Gene Ther 8: 1762-1769, 2001.

14. Smith PG, Oakley F, Fernandez M, Mann DA, Lemoine NR and Whitehouse A: Herpesvirus saimiri-based vector biodistribution using noninvasive optical imaging. Gene Ther 12: 1465-1476, 2005.

15. Macnab S, White R, Hiscox J and Whitehouse A: Production of an infectious Herpesvirus saimiri-based episomally maintained amplicon system. J Biotechnol 134: 287-296, 2008.

16. Hong Y, Macnab S, Lambert LA, Turner AJ, Whitehouse A and Usmani BA: Herpesvirus saimiri-based endothelin-converting enzyme-1 shRNA expression decreases prostate cancer cell invasion and migration. Int J Cancer 129: 586-598, 2011.

17. Macnab SA, Turrell SJ, Carr IM, Markham AF, Coletta PL and Whitehouse A: Herpesvirus saimiri-mediated delivery of the adenomatous polyposis coli tumour suppressor gene reduces proliferation of colorectal cancer cells. Int J Oncol 39: 1173-1181, 2011.

18. Hiller C, Tamguney G, Stolte N, et al: Herpesvirus saimiri pathogenicity enhanced by thymidine kinase of herpes simplex virus. Virology 278: 445-455, 2000.

19. Hiller C, Wittmann S, Slavin S and Fickenscher H: Functional long-term thymidine kinase suicide gene expression in human T cells using a herpesvirus saimiri vector. Gene Ther 7: 664-674, 2000.

20. Knappe A, Feldmann G, Dittmer U, et al: Herpesvirus saimiritransformed macaque $\mathrm{T}$ cells are tolerated and do not cause lymphoma after autologous reinfusion. Blood 95: 3256-3261, 2000 . 
21. Wiley SR, Schooley K, Smolak PJ, et al: Identification and characterization of a new member of the TNF family that induces apoptosis. Immunity 3: 673, 1995.

22. Pan G, Ni J, Wei Y-F, Yu G-1, Gentz R and Dixit VM: An antagonist decoy receptor and a death domain-containing receptor for TRAIL. Science 277: 815-818, 1997.

23. Walczak H, Degli-Esposti MA, Johnson RS, et al: TRAIL-R2: a novel apoptosis-mediating receptor for TRAIL. EMBO J 16 5386-5397, 1997.

24. Zhang L and Fang B: Mechanisms of resistance to TRAILinduced apoptosis in cancer. Cancer Gene Ther 12: 228, 2004.

25. Yamanaka T, Shiraki K, Sugimoto K, et al: Chemotherapeutic agents augment TRAIL-induced apoptosis in human hepatocellular carcinoma cell lines. Hepatology 32: 482-490, 2000.

26. Brooks AD, Jacobsen KM, Li W, Shanker A and Sayers TJ: Bortezomib sensitizes human renal cell carcinomas to TRAIL apoptosis through increased activation of caspase- 8 in the death-inducing signaling complex. Mol Cancer Res 8: 729-738, 2010.

27. Mori T, Doi R, Kida A, et al: Effect of the XIAP inhibitor embelin on TRAIL-induced apoptosis of pancreatic cancer cells. J Surg Res 142: 281, 2007.

28. Kelly MM, Hoel BD and Voelkel-Johnson C: Doxorubicin pretreatment sensitizes prostate cancer cell lines to TRAIL induced apoptosis which correlates with the loss of c-FLIP expression. Cancer Biol Ther 1: 520-527 2002.

29. White RE, Calderwood MA and Whitehouse A: Generation and precise modification of a herpesvirus saimiri bacterial artificial chromosome demonstrates that the terminal repeats are required for both virus production and episomal persistence. J Gen Virol 84: 3393-3403, 2003.

30. Turrell SJ and Whitehouse A: Mutation of herpesvirus Saimiri ORF51 glycoprotein specifically targets infectivity to hepatocellular carcinoma cell lines. J Biomed Biotechnol 2011: 785158, 2011.

31. Schmittgen TD and Livak KJ: Analyzing real-time PCR data by the comparative $C_{T}$ method. Nat Protocols 3: 1101-1108, 2008.

32. Thome M, Schneider P, Hofmann K, et al: Viral FLICEinhibitory proteins (FLIPs) prevent apoptosis induced by death receptors. Nature 386: 517-521, 1997.

33. Stevenson AJ, Giles MS, Hall KT, et al: Specific oncolytic activity of herpesvirus saimiri in pancreatic cancer cells. Br J Cancer 83: 329-332, 2000.
34. Ivanov VN, Partridge MA, Huang SXL and Hei TK: Suppression of the proinflammatory response of metastatic melanoma cells increases TRAIL-induced apoptosis. J Cell Biochem 112: 463-475, 2011.

35. Haura E, Turkson J and Jove R: Mechanisms of disease: insights into the emerging role of signal transducers and activators of transcription in cancer. Nat Rev Clin Oncol 2: 315-324, 2005.

36. Kanda N, Seno H, Konda Y, et al: STAT3 is constitutively activated and supports cell survival in association with survivin expression in gastric cancer cells. Oncogene 23: 4921-4929, 2004.

37. Fuke H, Shiraki K, Sugimoto K, et al: Jak inhibitor induces $\mathrm{S}$ phase cell-cycle arrest and augments TRAIL-induced apoptosis in human hepatocellular carcinoma cells. Biochem Biophys Res Commun 363: 738-744, 2007.

38. Newsom-Davis T, Prieske $\mathrm{S}$ and Walczak H: Is TRAIL the holy grail of cancer therapy? Apoptosis 14: 607-623, 2009.

39. Herbst RS, Eckhardt SG, Kurzrock R, et al: Phase I doseescalation study of recombinant human Apo2L/TRAIL, a dual proapoptotic receptor agonist, in patients with advanced cancer. J Clin Oncol 28: 2839-2846, 2010.

40. Soria J-C, Smit E, Khayat D, et al: Phase 1b sudy of dulanermin (recombinant human Apo2L/TRAIL) in combination with paclitaxel, carboplatin, and bevacizumab in patients with advanced non-squamous non-small-cell lung cancer. J Clin Oncol 28: $1527-1533,2010$

41. He C, Lao W-F, Hu X-T, Xu X-M, Xu J and Fang B-L: Anti-liver cancer activity of TNF-related apoptosis-inducing ligand gene and its bystander effects. World J Gastroenterol 10: 654-659, 2004

42. Niu G, Bowman T, Huang M, et al: Roles of activated Src and Stat3 signaling in melanoma tumor cell growth. Oncogene 21: 7001-7010, 2002

43. Ambrosini G, Adida C and Altieri D: A novel anti-apoptosis gene, survivin, expressed in cancer and lymphoma. Nat Med 3: 917-921, 1997.

44. Li F, Ambrosini G, Chu EY, et al: Control of apoptosis and mitotic spindle checkpoint by survivin. Nature 396: 580-584, 1998.

45. Shin S, Sung B-J, Cho Y-S, et al: An anti-apoptotic protein human survivin is a direct inhibitor of caspase-3 and -7 . Biochemistry 40: 1117-1123, 2001.

46. Sun C, Nettesheim D, Liu Z and Olejniczak ET: Solution structure of human survivin and its binding interface with Smac/Diablo. Biochemistry 44: 11-17, 2004. 